\title{
Revisión de criterios de sostenibilidad en muros de concreto para viviendas sismorresistentes
}

\author{
Revision of Sustainable Criteria of Concrete Walls for \\ Earthquake-Resistant Housing
}

\author{
Carrillo J. \\ Departamento de Ingeniería Civil \\ Universidad Militar Nueva Granada, UMNG, Bogotá, Colombia \\ Alcocer S.M. \\ Universidad Nacional Autónoma de México, UNAM \\ Correo:salcocerm@ii.unam.mx
}

Correo:wjcarrillo@gmail.com

Información del artículo: recibido: junio de 2011, reevaluado: diciembre de 2011, aceptado: marzo de 2012

\section{Resumen}

El desempeño sísmico de viviendas de baja altura ha sido notoriamente deficiente durante los últimos sismos ocurridos en países latinoamericanos. Adicionalmente, la revisión de la literatura reveló que algunas técnicas de construcción tradicionales no contribuyen a la construcción de viviendas sostenibles. En la última década, la construcción de viviendas con muros de concreto se ha convertido en una opción preferida debido a la velocidad de construcción y la disponibilidad de materiales en la mayoría de estos países. Con el propósito de mejorar los métodos de diseño sísmico para este tipo de construcción, se llevó a cabo un extenso programa de investigación, experimental y analítico. El programa experimental incluyó ensayos cuasi-estáticos y en mesa vibradora de 47 muros con diferentes relaciones altura-longitud y muros con aberturas. Las variables estudiadas fueron el tipo de concreto, la cuantía de acero de refuerzo en el alma y el tipo de acero de refuerzo para proveer dicha cuantía. El artículo presenta y analiza los resultados principales del programa de investigación y evalúa la factibilidad técnica y ambiental para utilizar muros de concreto en viviendas sostenibles y sismorresistentes. El desempeño de la vivienda con muros de concreto se evalúa en términos de los principales requerimientos ambientales y de resistencia sísmica. Se encontró que la vivienda a base de muros de concreto no sólo es segura ante sismos y fácilmente adaptable a diferentes ambientes, sino que incentiva la conservación del medio ambiente y promueve la reducción de los costos de construcción, operación y mantenimiento.

\section{Descriptores:}

- vivienda de interés social

- desarrollo sostenible

- diseño sismorresistente

- concreto ligero

- concreto reforzado con fibras de acero 


\begin{abstract}
The seismic performance of low-rise housing has been noticeably inadequate during the most recent earthquakes occurred in Latin American countries. Moreover, the literature review revealed that some traditional techniques do not contribute to building sustainable housing. In the last decade, construction of concrete walls housing has become a preferred choice because of the speed of construction and availability of materials in most of these countries. Aimed at improving seismic design methods for this type of construction, an extensive analytical and experimental program was carried out. The experimental program included quasi-static and shakingtable tests of 47 walls with different height-to-length ratios and walls with openings. Variables studied were type of concrete, web steel ratio and type of web reinforcement. The paper presents and discusses the main results of the research program and evaluates the technical and environmental feasibility for using concrete walls for sustainable and earthquake-resistant housing. Performance of concrete walls housing is assessed in terms of key environmental and earthquake-resistant requirements. It was found that concrete wall housing is not only safe under earthquakes and easily adaptable to climate, but also it stimulates environmental conservation and promotes reducing the costs of construction, operation and maintenance.
\end{abstract}

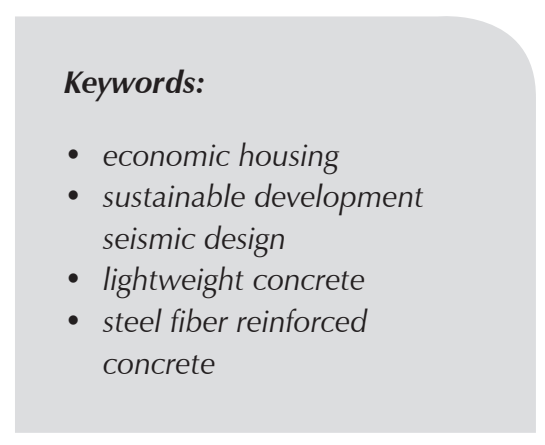

\section{Introducción}

El objetivo de la vivienda sostenible es ofrecer mejoras en aspectos relacionados con las emisiones de dióxido de carbono y el uso del agua (Lodge, 2009), pero sin comprometer el diseño estructural o la calidad de la construcción. A partir de un análisis del panorama actual de la vivienda, se destaca la necesidad de desarrollar técnicas de construcción integrales que garanticen la seguridad estructural y un desarrollo urbano sostenible. Para implantar un plan de vivienda sostenible, inicialmente se deben superar varios obstáculos que incluyen, entre otras cosas, dar un giro a la tendencia histórica en la edificación de vivienda, promover los beneficios ambientales y de calidad de vida que implica la adopción de técnicas de construcción que sean amigables con el medio ambiente, establecer especificaciones detalladas de construcción de los nuevos materiales y sistemas constructivos, e integrar a los desarrolladores inmobiliarios del país para que construyan bajo estas consideraciones.

La demanda de mayor cantidad de viviendas a bajo costo ha llevado a probar nuevas técnicas para la construcción de vivienda. La entrada al mercado de nuevos materiales y productos es una opción para construir de manera diferente, por ejemplo, el concreto ligero ofrece propiedades térmicas que promueven ahorro de energía para el usuario, adecuadas propiedades acústicas y de resistencia al fuego, así como la reducción de cargas muertas en las estructuras (CEMEX, 2011). Por otro lado, aunque las fibras más utilizadas para reforzar el concreto son las fibras de acero, vidrio, plástico, sintéticas y poliméricas, las fibras naturales se pueden obtener a un bajo costo usando la mano de obra disponible en la localidad y las técnicas adecuadas para su obtención. En el ámbito mundial, los países investigan sus propias fibras: de coco en Brasil, de palma en Egipto y Asia, de bambú en algunos países latinoamericanos y las de la familia del agave en México (Juárez et al., 2004).

En cuanto a la seguridad estructural, el desempeño de viviendas de baja altura ha sido notoriamente deficiente durante los últimos sismos ocurridos en Colombia, Haití, México y Perú. La ausencia de reglamentos adecuados para el diseño de vivienda es la causa principal de esta tendencia (Carrillo y Alcocer, 2011). Actualmente, la construcción de viviendas con muros de concreto es una de las opciones integralmente eficientes, es decir, satisface los requisitos sismorresistentes y puede ser ambientalmente amigable con el planeta. Con el propósito de incrementar y mejorar la oferta tecnológica de las viviendas de interés social construidas con muros de concreto, se llevó a cabo un extenso programa de investigación experimental y analítico, a partir del cual se desarrollaron recomendaciones para construcción, análisis y diseño sismorresistente. En este artículo se discuten los resultados principales de la investigación y se evalúa el diseño de la vivienda con muros de concreto desde una perspectiva integral, es decir, se discute el cumplimiento de los mínimos requisitos ambientales y de seguridad estructural. 


\section{Desarrollo sostenible}

El concepto de desarrollo sostenible que se plantea en el documento denominado: "Nuestro futuro común", es quizás el más difundido y aceptado a nivel internacional. En dicho documento, el desarrollo sostenible se define como "aquel desarrollo que satisface las necesidades presentes sin comprometer las opciones de las necesidades futuras", es decir, no agotar ni desperdiciar los recursos naturales y tampoco lesionar el medio ambiente ni a los seres humanos. Como se desprende de dicho concepto, no se pretende que no se utilicen los recursos, sino que se dé uso coherente de los mismos. Esta coherencia consiste en compatibilizar el progreso económico con las necesidades sociales y medioambientales que configuran el bienestar de los ciudadanos (López et al., 2005; González, 2003). En este mismo sentido, las viviendas sostenibles son casas ambientalmente amigables con el planeta. Este tipo de estructuras utilizan menos energía, producen menos desechos, son más saludables medioambientalmente para las personas que las habi$\tan y$, por lo tanto, generan ahorros económicos importantes.

Tendencias de la planeación urbana

La industria de la construcción es responsable de $40 \%$ del consumo mundial de energía eléctrica, 30\% de los gases nocivos y un alto porcentaje de desechos sólidos (Wolpert, 2008). Entre las tendencias principales en la búsqueda de soluciones urbanas más sostenibles en el mundo de hoy se destacan las siguientes (González, 2003):

- El incremento de las densidades del desarrollo urbano para lograr un mejor uso del suelo y la conservación del terreno verde.

- El reciclamiento de los recursos, incluyendo el agua, los residuos líquidos y sólidos, tanto orgánicos como inorgánicos, así como los materiales y elementos de construcción.

- La reducción del consumo de energía convencional a partir del diseño bioclimático, la eficiencia energética y el aprovechamiento de fuentes renovables de energía.

Reglamentos y acreditación para viviendas sostenibles

A finales del año 2006, se introdujo como reglamento voluntario en el Reino Unido "El reglamento para viviendas sostenibles" (DCLG, 2006), y a inicio de 2008 se esta- bleció como reglamento tanto en el Reino Unido como en los Estados Unidos. El reglamento califica seis elementos claves del diseño y construcción que impactan la sostenibilidad y la eficiencia, y debe ser utilizado por arquitectos, constructores y consumidores como método de ayuda para la planeación y el diseño de nuevas viviendas.

El reglamento asigna a la vivienda una puntuación con base en su desempeño medido a partir de los siguientes criterios de sostenibilidad: emisiones de $\mathrm{CO}_{2}$, agua, fugas de agua, materiales, desechos, contaminación, salubridad y buena apariencia, manejo de los impactos ambientales de la construcción y operación además de la ecología.

En Australia, a partir de julio de 2005, el gobierno estableció el índice de sostenibilidad del edificio (BASIX, 2011) para todas las viviendas nuevas de Sydney. BASIX es una nueva herramienta de planeación que exige a los nuevos desarrollos residenciales reducir el consumo de agua y energía en $40 \%$, en comparación con una vivienda promedio. Del mismo modo, la acreditación de liderazgo en energía y diseño ambiental (LEED por sus siglas en inglés) promueve la adopción global de prácticas de desarrollo de la construcción ecológica y sostenible, a través de la creación y aceptación de estándares, herramientas y criterios de rendimiento universalmente entendidos y aceptados. Los criterios de evaluación LEED incluyen: la eficacia energética y del consumo de agua, la utilización de materiales de procedencia local y el reciclamiento de sus desperdicios (Agudo, 2008).

\section{Nuevos materiales para construcción de muros para viviendas}

La demanda de mayor cantidad de viviendas a bajo costo ha llevado a probar nuevas técnicas para la construcción de vivienda. La entrada al mercado de nuevos materiales y productos es una opción para construir de manera diferente.

En Estados Unidos y Europa, gran parte de las construcciones se lleva a cabo con nuevos materiales, a diferencia de la mayoría de países latinoamericanos, en donde sigue prevaleciendo el tabique como el material con mayor demanda. A continuación, se describen algunos materiales con gran potencial para la construcción de viviendas de interés social.

\section{Concreto ligero}

Las principales ventajas del concreto ligero para la construcción de vivienda son (CEMEX, 2011): 
a) Propiedades térmicas eficientes que promueven ahorro de energía para el usuario.

b) Adecuadas propiedades acústicas y de resistencia al fuego.

c) Permite reducir las cargas muertas en las estructuras $\mathrm{y}$, de esta manera, se reducen las fuerzas sísmicas.

d) Su alta manejabilidad favorece las operaciones de colocación y elimina la utilización de vibradores, por lo tanto, reduce los costos de construcción, disminuye la permeabilidad.

El uso del concreto ligero en fachadas expuestas es especialmente recomendable para muros orientados este y oeste, ya que este concreto tiene poca resistencia térmica (Adarve, 2002).

\section{Concreto reforzado con fibras}

El concreto simple (sin refuerzo) tiene baja resistencia a la tensión y capacidad limitada de deformación a la fa1la. Tradicionalmente, para mejorar este comportamiento se adicionan barras de refuerzo o acero de preesfuerzo. El acero de refuerzo es continuo y está localizado en un sitio específico para mejorar el desempeño de la estructura. En cambio, las fibras son discontinuas y generalmente están distribuidas de forma aleatoria en la matriz de concreto. Actualmente, las fibras se utilizan junto con el refuerzo convencional para aplicaciones estructurales (ACI-544.1R, 2002). Debido a la flexibilidad de los métodos de fabricación y el adecuado desempeño a flexión, el Concreto Reforzado con Fibras de Acero (CRFA) puede ser un material económico y versátil para la construcción de vivienda. Por ejemplo,

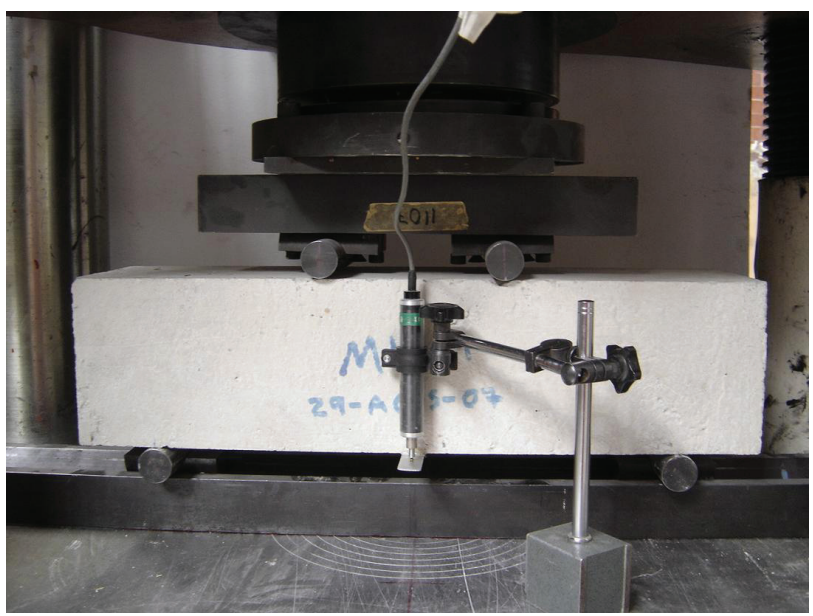

a) Configuración de ensayo

Figura 1. Comportamiento a flexión del CRFA en la figura 1 se observan la configuración del ensayo y el desempeño a flexión medido en concretos con y sin fibras de acero de este estudio.

Las fibras más utilizadas para reforzar el concreto son las fibras de acero (figura 2), vidrio, plástico, sintéticas y poliméricas. Todas tienen sus ventajas y desventajas, por ejemplo, el vidrio sufre deterioro por la alcalinidad de la matriz del concreto, el acero puede sufrir corrosión, los plásticos son poco elásticos, etcétera. Existe otro grupo conocido como fibras naturales no procesadas, las cuales han sido motivo de diversos estudios para su posible aplicación en el reforzamiento del concreto. Los materiales reforzados con fibras naturales se pueden obtener a un bajo costo usando la mano de obra disponible en la localidad y las técnicas adecuadas para su obtención. Se ha hecho una gran inversión en la investigación de las fibras naturales para darle aplicación industrial y para estudiar la durabilidad del concreto con este tipo de fibras. En el ámbito mundial los países investigan sus propias fibras: de coco en Brasil, palma en Egipto y Asia, de bambú en algunos países latinoamericanos, y las de la familia del agave en México (Juárez et al., 2004).

\section{Desempeño de viviendas construidas con muros de concreto}

El colapso parcial o total de un número significativo de viviendas durante los sismos ocurridos en Colombia, Haití, México y Perú, nuevamente ha evidenciado la ausencia de recomendaciones y reglamentos de diseño eficientes para la construcción de viviendas de baja altura. Desafortunadamente, la población con recursos económicos limitados resulta ser la más afectada tras la

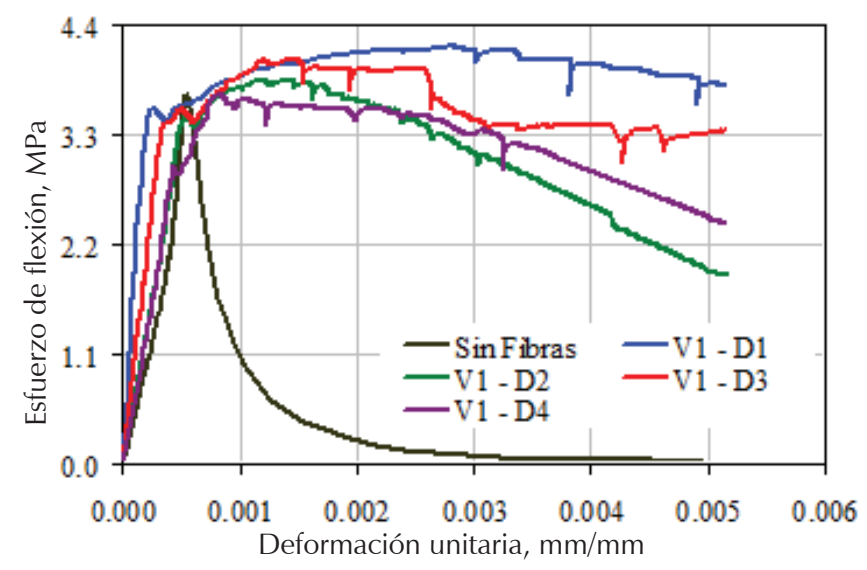

b) Curva carga-deformación 


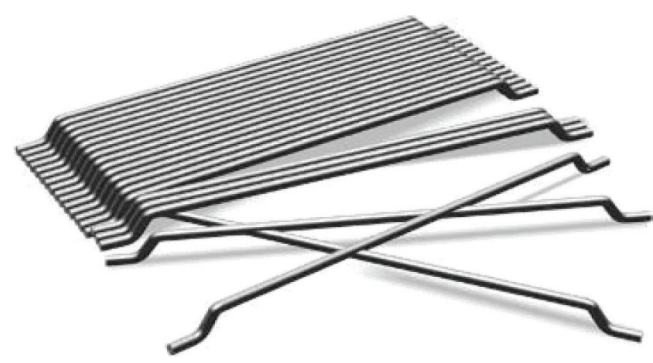

a) Con gancho

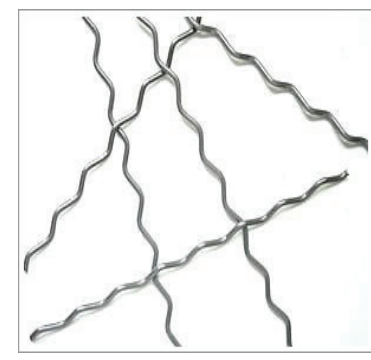

b) Onduladas

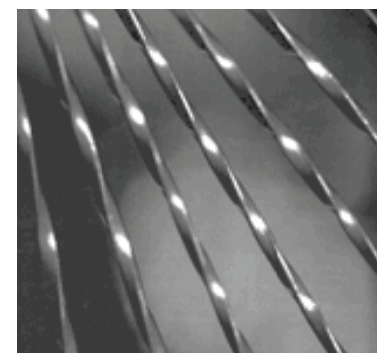

c) Retorcidas

Figura 2. Principales tipos de fibras de acero

ocurrencia de desastres naturales. Una de las opciones más eficientes para la construcción de viviendas de interés social es el desarrollo de conjuntos habitacionales con viviendas de concreto en su totalidad (cimentación, muros, losas de entrepiso y techos).

A partir de un diseño sísmico basado en el desempeño, es posible proporcionar a la vivienda las características necesarias de acuerdo con el tipo de materiales empleados, la geometría de la estructura y la demanda sísmica sobre los elementos, por ejemplo, considerando la resistencia inherente de estructuras con muros de concreto en viviendas de baja altura, se pueden utilizar muros con resistencia de concreto baja y espesor reducido. Adicionalmente, en zonas donde las demandas sísmicas no son una preocupación en el diseño estructural, el refuerzo mínimo a cortante estipulado en los reglamentos NTC-C (2004) y ACI-318 (2008), parece ser excesivo para controlar el agrietamiento por tensión diagonal en los muros.

\section{Programa experimental para evaluar el desempeño sísmico}

Con el propósito de incrementar y mejorar la oferta tecnológica de las viviendas de interés social construidas con muros de concreto, en el Instituto de Ingeniería de la UNAM se desarrolló un extenso programa de investigación experimental y analítico, el cual se dividió básicamente en tres etapas: en la primera se estudió el comportamiento de 39 muros con refuerzo convencional (barras corrugadas y malla de alambre soldado), en la segunda, se evaluó la técnica de rehabilitación de 2 muros utilizando CRFA y, en la tercera, se estudió el desempeño de 6 muros reforzados a cortante en el alma utilizando exclusivamente CRFA. El objetivo general de la investigación fue proponer criterios de análisis y diseño sísmico aplicables a muros de concreto para viviendas de baja altura.

\section{Muros con refuerzo convencional}

El programa experimental de la primera etapa incluyó 39 ensayos cuasi-estáticos y dinámicos de muros con diferente relación de aspecto y sistemas de muros con aberturas (Flores et al., 2007; Carrillo, 2010; Sánchez, 2010).

Las variables de estudio se obtuvieron de las empleadas con más frecuencia en la práctica del diseño y construcción de viviendas de concreto en Latinoamérica (tabla 1).

Tabla 1. Descripción de las variables de la etapa 1

\begin{tabular}{|c|c|}
\hline Variable & Descripción \\
\hline $\begin{array}{l}\text { Relación de aspecto } \\
(h w / l w):\end{array}$ & $\begin{array}{l}\text { Cuadrados }\left(h_{w} / l_{w}=1\right) \text {, robustos }\left(h_{w} / l_{w}=0.5\right) \text {, esbeltos }\left(h_{w} / l_{w}=2\right) \text { y muros con aberturas (puerta y ventana). El } \\
\text { espesor }\left(t_{w}\right) \text { y la altura libre nominal }\left(h_{w}\right) \text { de todos los muros (en escala natural) fue igual a } 100 \mathrm{~mm} \text { y } 2.4 \mathrm{~m} \text {, } \\
\text { respectivamente. }\end{array}$ \\
\hline Tipo de concreto & $\begin{array}{l}\text { de peso normal, peso ligero y autocompactable, con resistencia nominal a la compresión, } f_{c}^{\prime}=15 \mathrm{MPa} \text {. Las } \\
\text { resistencias medidas variaron entre } 15 \text { y } 25 \mathrm{MPa} \text {, aproximadamente }\end{array}$ \\
\hline $\begin{array}{l}\text { Cuantía de refuerzo a } \\
\text { cortante en el alma } \\
\text { (vertical y horizontal) }\end{array}$ & $\begin{array}{l}100 \% \rho_{\min }(0.25 \%), 50 \% \rho_{\min }(0.125 \%), 0=\text { sin refuerzo en el alma. El porcentaje de la cuantía se expresó como } \\
\text { fracción de la cuantía mínima }\left(\rho_{\min }\right) \text { estipulada en los reglamentos de diseño NTC-C (2004) y ACI-318 (2008). } \\
\text { En todos los muros se proporcionó suficiente refuerzo en los extremos para prevenir falla por flexión }\end{array}$ \\
\hline \multicolumn{2}{|c|}{$\begin{array}{l}\text { Tipo de refuerzo a cortante Barras corrugadas de acero con esfuerzo de fluencia nominal, } f_{y}=412 \mathrm{MPa} \text { y malla de alambre soldado con } \\
\text { en el alma } \\
\text { esfuerzo de fluencia nominal, } f_{y}=491 \mathrm{MPa}\end{array}$} \\
\hline Tipo de ensayo & cuasi-estático monótono, cuasi-estático cíclico-reversible y dinámico en mesa vibradora \\
\hline
\end{tabular}


A partir del análisis de la información experimental y analítica se comprobó que las cuantías mínimas de refuerzo a cortante, estipuladas en los reglamentos para diseño sísmico de viviendas de baja altura son conservadoras o muy conservadoras, especialmente para estructuras situadas en algunas zonas de amenaza sísmica baja o moderada (Carrillo, 2010). Con base en lo anterior, Carrillo y Alcocer (2011) proponen recomendaciones de acuerdo con la capacidad y la demanda de las viviendas, por ejemplo, en algunas zonas se propuso prescindir o disminuir el refuerzo a cortante en el alma del muro a cambio de utilizar requisitos específicos por cambios volumétricos y/o refuerzo por integridad estructural, así como parámetros particulares para diseño sísmico.

\section{Muros reforzados con fibras de acero}

Estudios experimentales previos (Kwak et al., 2002; Parra, 2005) han indicado que el concreto reforzado con fibras de acero (CRFA) incrementa la resistencia a esfuerzo cortante y la capacidad de deformación de elementos estructurales. En el programa experimental de la etapa 2 se estudió el comportamiento dinámico de dos muros de concreto de baja altura rehabilitados mediante un encamisado de CRFA (Ávila et al., 2011). En términos generales, la respuesta medida de los muros rehabilitados fue satisfactoria, ya que se registraron resistencias superiores y desplazamientos similares a los medidos en los muros originales. A partir del desempeño observado en vigas de concreto reforzado con fibras, el Reglamento ACI-318 (2008) permite el uso de fibras de acero en sustitución del acero de refuerzo mínimo por cortante en vigas, el cual se dispone de forma convencional utilizando estribos de acero. El ACI-318 permite dicha sustitución, siempre y cuando la resistencia nominal a la compresión del concreto $\left(f_{c}^{\prime}\right)$ sea menor que $40 \mathrm{MPa}$, el peralte de la viga sea menor que $60 \mathrm{~cm}$ y el esfuerzo cortante de diseño sea menor que $\phi 0.17 \sqrt{ } f_{c}^{\prime}$ MPa.

Considerando las características particulares de los muros de concreto para vivienda de baja altura y las demandas sísmicas en este tipo de estructuras, las propiedades mecánicas de los concretos con fibras, en es- pecial su resistencia a tensión y su capacidad de deformación post-agrietamiento, los hacen materiales idóneos para su utilización en la construcción de muros de concreto para vivienda. Sin embargo, las NTC-C (2004) no hacen referencia al uso de concreto reforzado con fibras. Por lo tanto, en el programa de investigación experimental de la etapa 3 se evaluó la factibilidad técnica y económica, así como el desempeño estructural de muros de concreto reforzados en el alma utilizando exclusivamente fibras de acero. En esta etapa se realizó el ensayo de seis muros de concreto con relación de aspecto igual a uno, que se construyeron utilizando concreto reforzado con diferentes tipos y dosificaciones de fibras de acero. De acuerdo con el espesor reducido de los muros y las especificaciones del ACI-318 (2008), se seleccionaron dos tipos de fibra de acero con gancho (figura 2a) y tres dosificaciones distintas. En la tabla 2 se describen las variables principales de la etapa 3. A partir del análisis de la información experimental, Alcocer et al. (2011) proponen los valores de dosificación mínima de fibras para sustituir el refuerzo convencional a cortante en muros de concreto para vivienda de baja altura.

\section{Evaluación de las características ambientales}

Para satisfacer la demanda de vivienda es necesario proporcionar soluciones innovadoras que optimicen los procedimientos y los costos de construcción, de esta manera, ofrecer viviendas de mejor calidad a los consumidores finales sin afectar el confort y el precio de venta (Uribe, 2007). Si bien el costo unitario de los materiales empleados en los muros de concreto es superior al de la mampostería tradicional, el ahorro económico y la edificación sostenible se logran con la rapidez de construcción y el uso de concretos de características especiales. Por ejemplo, la utilización de sistemas industriales de cimbras de acero así como mallas de alambre soldado como refuerzo a cortante en el alma garantiza la disminución del tiempo y el costo de la vivienda. El empleo de concretos de tipo ligero y autocompactable también promueve el ahorro de energía y prolonga la vida útil de las viviendas. Adicionalmente, cuando se reemplaza la malla de alambre soldado y el

\begin{tabular}{lllll}
\hline \multicolumn{1}{c}{ Variable } & \multicolumn{5}{c}{ Descripción de la variable } \\
\hline \multirow{3}{*}{ Fibra de acero } & Referencia & RC-65/35-BN & RC-80/60-BN \\
& Longitud, $l_{f}(\mathrm{~mm})$ & 35 & 60 & \\
& Diámetro, $d_{f}(\mathrm{~mm})$ & 0.55 & 0.75 & \\
& Relación de aspecto $\left(l_{f} / d_{f}\right)$ & 64 & 80 & 75 \\
\hline Dosificación & $\mathrm{D}_{f}\left(\mathrm{~kg} / \mathrm{m}^{3}\right)$ & 45 & 60 & 7 \\
\hline
\end{tabular}

Tabla 2. Descripción de las variables de la etapa 3 
acero de refuerzo tradicional por fibras de acero, se logran reducir los costos de mano de obra (mayor velocidad de construcción) y de los materiales (reducción de espesor de los elementos).

Según Adarve (2002), los muros delgados de concreto pueden lograr buena adaptación al clima, ya que la densidad y capacidad de reflexión solar (color exterior claro) son los factores principales que inciden en el balance térmico de la construcción. En cuanto a los tipos de muros, Adarve (2002) ha recomendado diferentes tipos de muros y losas de concreto que aseguran buena eficiencia termodinámica para diferentes tipos de clima.

Para mejorar la competitividad del concreto en el mercado de los materiales sostenibles, se llevaron a cabo estudios para investigar la utilización de nuevos materiales de adición y de nuevas tecnologías de construcción. Los métodos recientemente desarrollados para la construcción de viviendas de concreto generan menos $\mathrm{CO}_{2}$ que algunos métodos tradicionales de construcción de viviendas. Las investigaciones han revelado que durante el promedio de vida de una vivienda de concreto (figura 3), las contribuciones de $\mathrm{CO}_{2}$ son menores que las generadas por viviendas a base de madera o mampostería tradicional. Aunque la manufactura inicial del cemento es de energía intensiva, estos sistemas de muros de excelente desempeño necesitan menos energía para la calefacción y aire acondicionado de cada día, por ejemplo, en aproximadamente cinco a siete años, el valor total de $\mathrm{CO}_{2}$ de una vivienda típica de muros de madera empieza a exceder al de una vivienda de concreto. Este beneficio ambiental continúa por todo el tiempo en que la casa es utilizada. Adicionalmente, el concreto contribuye a la calidad del aire en el interior, ya que los nuevos concretos no producen fugas, como es frecuente con muchos otros materiales de construcción (PCA, 2005).

Aunado a que el concreto tiene uno de los periodos de vida útil más largos entre los materiales de construc-

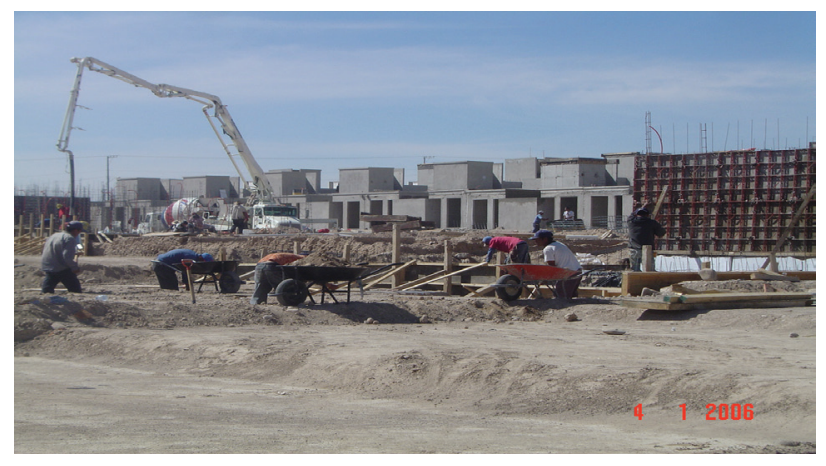

Figura 3. Vivienda de concreto sostenible y sismorresistente ción, su utilidad no termina después de su propósito original. En la mayoría de las áreas urbanas, casi todo el concreto puede ser triturado y reciclado para usarse como base en las carreteras y como terraplén. En algunos casos, el concreto se puede reciclar para servir como agregado de concreto nuevo. El reciclamiento de materiales es completamente amigable con el medio ambiente, ya que reduce el consumo de energía, disminuye el desecho y aumenta la vida de los materiales. Es importante que los constructores planeen reducir, reutilizar y reciclar los desechos de las construcciones. Los constructores se darán cuenta que pierden dinero cuando incrementen los costos de los rellenos sanitarios y comience a aplicarse estrictamente la normatividad de disposición de residuos sólidos. Si se actúa ahora, los constructores también pueden comenzar a estimular el mercado necesario para el manejo de sus materiales de desecho. Actualmente, estos mercados son débiles o simplemente, no existen (Walther, 1993). Por lo tanto, además de las aplicaciones de reciclamiento indicadas, la investigación debe continuar estudiando y encontrando nuevas aplicaciones para el concreto reciclado.

\section{Conclusiones}

Para la construcción de viviendas en algunos casos se utilizan técnicas que se basan en criterios exclusivamente ambientales; sin embargo, todas las técnicas de construcción deben ser integrales, es decir, tanto el desempeño estructural como ambiental de la vivienda debe ser satisfactorio. Por lo tanto, se recomienda que los reglamentos establezcan recomendaciones para el análisis, diseño, construcción y evaluación, junto con especificaciones y métodos de ensayo para verificar el comportamiento sísmico, el desempeño medioambiental (incluyendo los efectos de humedad y durabilidad), la resistencia al fuego, así como las propiedades acústicas y de aislamiento de todos los sistemas constructivos

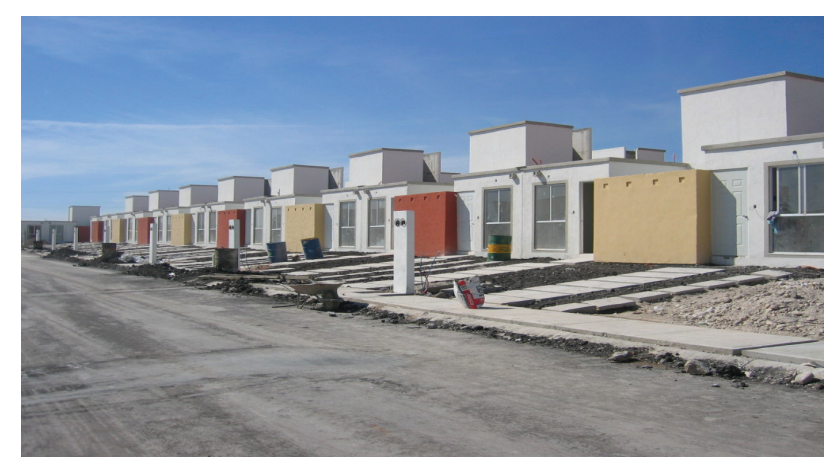


que se utilicen para la construcción de viviendas. Estas recomendaciones son importantes para brindar seguridad, economía y confort al usuario final de la vivienda, así como para cumplir los requisitos ambientales de construcción.

Con el propósito de promover la construcción de una vivienda sostenible y sismorresistente, en este estudio se evaluó la factibilidad técnica para la utilización de muros de concreto. A partir del análisis de la información disponible se comprobó que es posible proporcionar a la vivienda de concreto las características sismorresistentes necesarias de acuerdo con el tipo de materiales empleados, la geometría de la estructura y la demanda sísmica sobre los muros. Aunque el costo unitario de los materiales empleados en los muros de concreto es superior al de la mampostería tradicional con tabiques, el ahorro económico y la edificación sostenible se logran con la rapidez de construcción que brinda la utilización de muros delgados fácilmente adaptables al clima, cimbras industriales y mallas de alambre soldado, con el uso de concretos de características especiales (ligero, autocompactable y CRFA) y el reciclamiento del concreto para formar nuevos materiales. Por lo tanto, la vivienda a base de muros delgados de concreto aquí estudiada es segura ante eventos sísmicos, incentiva la conservación del medio ambiente y promueve la reducción de los costos de construcción, operación y mantenimiento.

\section{Agradecimientos}

Los autores agradecen al Grupo CEMEX por el apoyo económico de la investigación, así como al personal de los laboratorios del Instituto de Ingeniería de la UNAM, por su valiosa colaboración durante la realización de los ensayos. El contenido de este artículo representa exclusivamente la opinión de los autores y no refleja la opinión de los patrocinadores y colaboradores.

\section{Referencias}

Adarve A. Recomendaciones para el confort climático de las viviendas en concreto, en: ASOCRETO 2002, Cartagena, Colombia, 2002.

Alcocer S., Carrillo J. y Ávila O. Uso de concretos con fibras para muros de vivienda popular, informe técnico, Instituto de Ingeniería, UNAM, 2011, 62 p.

ACI-Comité 318. Building Code Requirements for Structural Concrete (ACI 318-08) and Commentary (ACI 318R-08), American Concrete Institute, Farmington Hills, MI, EUA, 2008.
ACI-Comité 544. State-of-the-Art Report on Fiber Reinforced Concrete (ACI 544.1R-96). American Concrete Institute, Farmington Hills, MI, EUA, 1996.

Agudo J. Desarrollo sustentable. Presentación: Su Casita, México, 2008 [en línea], [fecha de consulta: junio]. Disponible en: www.canadevivallemexico.org. $\mathrm{mx}$

Ávila O., Carrillo J. y Alcocer S. Rehabilitación de muros de concreto usando CRFA: Ensayos en mesa vibradora. Concreto y Cemento: Investigación y Desarrollo, IMCYC, volumen 2 (número 2), 2011: 2-17.

BASIX. Building Sustainable Index, 2011 [en línea], [fecha de consulta: mayo]. Disponible en: www.basix.nsw.gov.au

Carrillo J. Evaluación del comportamiento al cortante de muros de concreto para vivienda por medio de ensayos dinámicos, tesis (doctorado en ingeniería), México, DF, Universidad Nacional Autónoma de México, 2010, 474 p.

Carrillo J. y Alcocer S. Comportamiento a cortante de muros de concreto para vivienda. Ingeniería Sísmica, (volumen 85), julio-diciembre 2011: 103-126.

CEMEX. Concreto profesional-Ligero celular, 2011 [en línea], [fecha de consulta: mayo]. Disponible en: www.cemexmexico.com

DCLG-Department for Communities and Local Government. Code for Sustainable Homes: a Step-Change in Sustainable Home Building Practice, Londres, Reino Unido, 2006.

Flores L, Alcocer S, Carrillo J, Sánchez A, Uribe R y Ponce A. Ensaye de muros de concreto con diferente relación de aspecto y bajas cuantías de refuerzo, para uso en vivienda, en: XVI Congreso Nacional de Ingeniería Sísmica, IxtapaZihuatanejo, México, Tema XI, Artículo 2, 2007.

González D. Vivienda y sustentabilidad urbana: Conceptos y propuestas. Arquitectura y Urbanismo, volumen 24 (número 2), 2003: 34-42.

Juárez C., Váldez P. y Durán A. Fibras naturales de lechuguilla como refuerzo en materiales de construcción. Ingeniería de Construcción, volumen 19 (número 2), 2004: 83-92.

Kestner D, Goupil J y Lorenz E. Sustainability Guidelines for the Structural Engineer. American Society of Civil Engineers, ASCE, Reston, VA, Canadá, 2010.

Kwak Y., Eberhard M., Kim W. y Kim J. Shear Strength of Steel Fiber-Reinforced Concrete Beams without Stirrups. ACI Structural Journal, volumen 99 (número 4), 2002: 530-538.

Lodge R. Green Home Building for Eco-Friendly Living, 2009 [en línea], [fecha de consulta: junio]. Disponible en: www. designophy.com

López C., López E. y Peniche I. Desarrollo sustentable o sostenible: una definición conceptual. Horizonte Sanitario, volumen 4 (número 2), 2005. 
NTC-C Normas técnicas complementarias para diseño y construcción de estructuras de concreto, Gaceta Oficial del Distrito Federal, México DF, 2004, 101 p.

Parra-Montesinos G. High-Performance Fiber-Reinforced Cement Composites: an Alternative for Seismic Design of Structures. ACI Structural Journal, volumen 102 (número 5), 2005: 668-675.

PCA. Construyendo verde con concreto gris. Construcción y Tecnología, IMCYC, febrero de 2005.

Sánchez A. Comportamiento sísmico de viviendas construidas con muros de concreto, informe técnico, Instituto de Ingeniería, UNAM, México, DF, 2010, 475 p.

Uribe R. Viviendas sustentables, en: Centro de Tecnología de Cemento y Concreto. Aguascalientes, México, agosto de 2007.

Walther R. Builder's Guidebook to Reducing, Reusing and Recycling Residential Construction Waste in Wisconsin, enterprise center, University of Wisconsin, Madison, EUA, 1993.
Wolpert J. Aspectos de sustentabilidad en el diseño de vivienda, Comisión Nacional para el Ahorro de Energía-CONAE, Secretaría de Energía, México, 2008.

\section{Este artículo se cita:}

\section{Citación Chicago}

Carrillo, Julián, Sergio M. Alcocer. Revisión de criterios de sostenibilidad en muros de concreto para viviendas sismorresistentes. Ingeniería Investigación y Tecnología XIII, 04 (2012): 479-487.

\section{Citación ISO 690}

Carrillo J., Alcocer S.M. Revisión de criterios de sostenibilidad en muros de concreto para viviendas sismorresistentes. Ingeniería Investigación y Tecnología, volumen XIII (número 4), octubre-diciembre 2012: 479-487.

\section{Semblanza de los autores}

Julián Carrillo. Obtuvo los títulos de ingeniero civil en 2002 por la Universidad Militar Nueva Granada, UMNG, Colombia, el de maestro en ingeniería civil (área de estructuras y sísmica) en 2004 por la Universidad de los Andes, Colombia, y el de doctor en ingeniería (área de estructuras) en 2010 por la Universidad Nacional Autónoma de México, UNAM. Actualmente es profesor y director del Grupo de Investigación de Estructuras y Sísmica de la UMNG. Es miembro de tres comités del American Concrete Institute, ACI: 314, Diseño simplificado de edificios; 369, Reparación y rehabilitación sísmica; y 374, Diseño sísmico basado en desempeño de edificios de concreto.

Sergio M. Alcocer. Obtuvo los títulos de ingeniero civil en 1986 por la Universidad Nacional Autónoma de México, UNAM, y el de doctor en filosofía (área de estructuras) en 1991 por la Universidad de Texas en Austin, EUA. Actualmente es coordinador para innovación y desarrollo e investigador del Instituto de Ingeniería de la UNAM. Es miembro de cinco comités del American Concrete Institute, ACI: 318, Reglamento de construcción de concreto estructural; 352, Juntas y conexiones en estructuras monolíticas de concreto; 374, Diseño sísmico basado en desempeño de edificios de concreto; 445, Cortante y torsión; y 530, Normas de mampostería. 